\title{
Síndrome de cefaleia e défices neurológicos transitórios com linfocitose no LCR (HaNDL)
}

\author{
Syndrome of transient headache and neurologic deficits with cerebrospinal fluid \\ lymphocytosis (HaNDL)
}

Laryssa Crystinne Azevedo Almeida, Marcelo Moraes Valença

Unidade Funcional de Neurologia e Neurocirurgia, Universidade Federal de Pernambuco, Cidade Universitária, Recife, Pernambuco, Brasil

Almeida LCA, Valença MM. Síndrome de cefaleia e défices neurológicos transitórios com linfocitose no LCR (HaNDL) Headache Medicine. 2018;9(2):68-71

\begin{abstract}
RESUMO
Os autores fazem uma revisão narrativa sobre a "síndrome de cefaleia e défices neurológicos transitórios com linfocitose no LCR" (syndrome of transient headache and neurologic deficits with cerebrospinal fluid lymphocytosis, HaNDL), distúrbio raro, subdiagnosticado, de pouco conhecimento por médicos especializados no atendimento na emergência. São comentados aspectos do quadro clínico, diagnóstico, prognóstico e tratamento.
\end{abstract}

Palavras-chave: Enxaqueca; HaNDL; Cefaleia

\section{ABSTRACT}

The authors make a narrative review on "headache syndrome and transient neurological deficits with cerebrospinal fluid lymphocytosis (CRL)," a rare, underdiagnosed disorder of little knowledge by physicians specializing in care in the emergency room. Aspects of the clinical picture, diagnosis, prognosis, and treatment are discussed

Keywords: Migraine; HaNDL; Headache

\section{INTRODUÇÃO}

A síndrome de cefaleia e défices neurológicos transitórios com linfocitose no LCR é uma desordem rara, ${ }^{(1-4)}$ autolimitada e de curso benigno, que se caracteriza por episódios de cefaleia semelhante à migrânea, ${ }^{(5)}$ associadas a défices neurológicos e um aumento na contagem de linfócitos no LCR, apresentações essas que se resolvem espontaneamente em aproximadamente três meses. Já foi chamada como cefaleia pseudomigranosa ${ }^{(6-7)}$ pelas características semelhantes à migrânea. ${ }^{(8)}$ Encontra-se descrita na International Classification of Headache Disorders (ICHD - 3 beta - 2013)(9) como uma cefaleia secundária na categoria cefaleia atribuída a perturbação intracraniana não vascular.

\section{FISIOPATOLOGIA}

A síndrome foi primeiramente descrita na literatura em 1981 por Bartleson e colaboradores ${ }^{(5)}$ em uma série de sete pacientes como uma "desordem inflamatória subjacente do sistema nervoso central". Cerca de três décadas após a primeira descrição, a etiopatogenia exata do processo permanece desconhecida. Estudos de neuroimagem ${ }^{(10-17)}$ mostram um atraso na perfusão sem aumento da difusão e um estreitamente das artérias cerebrais durante as crises em áreas cerebrais correspondentes às manifestações clínicas. Estudos com ultrassonografia com Doppler transcraniano mostram alterações do fluxo de artérias intracranianas. ${ }^{(16,18)}$ 
Estudos usando EEG evidenciaram alterações elétricas do córtex cerebral ${ }^{(16)}$ tipicamente no hemisfério contralateral às manifestações neurológicas. ${ }^{(19-21)}$ Porém, as alterações eletroencefalográficas e no SPECT nem sempre se correlacionam com a topografia do déficit neurológico focal. ${ }^{(19)}$ Alterações no potencial evocado visual podem também ser encontradas. ${ }^{(22)}$ Muitos pacientes descrevem a apresentação de pródromos febris sugestivas de doenças virais, ${ }^{(23)}$ havendo descrição de detecção de partículas virais por meio do PCR do Herpes Virus ${ }^{(20,24)}$ tipos 6 e 7, anticorpos séricos IgM positivos para $C M V^{(7)}$ e o relato recente de autoanticorpos séricos para a subunidade do canal de cálcio tipo-T voltagem-dependente $(\mathrm{CACNA} 1 \mathrm{H}),{ }^{(25)}$ servindo como subsídios para reforçar a teoria de que uma autoimunidade ${ }^{(26)}$ esteja ligada ao processo desencadeante da desordem, porém mais estudos precisam ser desenvolvidos.

\section{APRESENTAÇÃO CLÍNICA}

É importante destacar que a maior parte dos pacientes com esta síndrome não apresenta história pregressa de migrânea. A maior série de casos, publicada (50 pacientes) por Gómez-Aranda e coautores, ${ }^{(4)}$ mostrou que a síndrome foi mais comum em homens (68\%), na idade entre 14 e 39 anos e apresentando uma média de três crises por paciente (variando entre 1 e 12 por paciente). Todos os pacientes apresentaram uma cefaleia de intensidade moderada a grave e a sintomatologia predominante foi: alterações sensitivas (66\%), seguida de fraqueza motora (42\%) e queixas visuais (18\%). Também foram descritos casos que apresentaram afasia, disartria e convulsões. Alterações da consciência, com estado de confusão mental

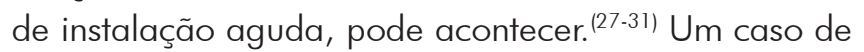
oftalmoplegia externa completa foi também descrito. ${ }^{(32)}$ Foi demonstrado que a síndrome pode cursar com aumento importante da pressão intracraniana, evidenciada por níveis altos na pressão do LCR, associada a clínica compatível: vômitos, papiledema ${ }^{(33)}$ e piora da intensidade da cefaleia. $\bigcirc$ episódio pode ser precipitado por angiografia cerebral. ${ }^{(34)}$ Na população pediátrica, ${ }^{(6,23,35-37)}$ a síndrome é incomum, mas é descrito na literatura alguns casos, cursando com apresentação clínica atípica, no contexto de um estado confusional agudo. ${ }^{(38)}$

\section{Diagnóstico}

O diagnóstico desta condição é eminentemente fundamentado em critérios clínicos e de alterações no LCR. A ICHD 3 beta $^{(9)}$ define os seguintes critérios diagnósticos:
A. Episódios de cefaleia de tipo enxaqueca preenchendo os critérios $B$ e $C$.

B. Os dois seguintes:

1. Acompanhados ou imediatamente precedidos do início de, pelo menos, um dos seguintes défices neurológicos transitórios, com duração $>4$ horas:

a) Hemi-hipostesia

b) Disfasia

c) Hemiparesia

2. Associada a pleocitose linfocitária (> 15 leucócitos $/ \mu \mathrm{L}$ ), com exames de neuroimagem normais, exame bacteriológico do LCR negativo e outros testes para investigação etiológica também negativos;

C. Evidência de causalidade demonstrada por um ou pelos dois seguintes:

1. O agravamento da cefaleia e dos défices neurológicos transitórios foram concomitantes ou levaram ao seu diagnóstico;

2. A melhoria da cefaleia e dos défices neurológicos transitórios foram concomitantes;

D. Não melhor explicada por outro diagnóstico da ICHD-3 beta.

É importante reforçar que a "síndrome de cefaleia e défices neurológicos transitórios com linfocitose no LCR" deve ser um diagnóstico de exclusão, (39) já que distúrbios mais graves e de consequências permanentes podem apresentar a mesma sintomatologia, como os acidentes vasculares encefálicos, ataques Isquêmicos transitórios ou meningoencefalites. Um caso de encefalite Anti-receptor de N-Methyl-D-Aspartate apresentou características clínicas muito sugestivas de HaNDL, com exceção dos anticorpos lgG no LCR do paciente. ${ }^{(39)}$ É válido ainda ressaltar que se deve atentar para o diagnóstico diferencial com outras cefaleias, como a migrânea hemiplégica familiar tipos 1, 2 ou 3 e migrânea hemiplégica esporádica, também descritas no ICHD- 3 beta como uma migrânea com aura incluído fraqueza motora, que tipicamente não cursa do pleocitose no LCR, mas raramente podem apresentá-la.

\section{Tratamento}

Diante do diagnóstico com base na exclusão de outras causas ${ }^{(0,33,40-41)}$ que não podem ter o seu tratamento adiado (uso de trombolíticos no AVEs, ${ }^{(17,}{ }^{42)}$ antibióticos nas meningoencefalites) evita-se que o paciente seja submetido a procedimentos invasivos para esclarecimento diagnóstico e internamento prolongado.

Entendendo-se a fisiopatologia da síndrome como um evento de hipoperfusão cerebral, alguns trabalhos defen- 
dem o uso de nimodipino e magnésio, estratégia semelhante à usada no vasoespasmo cerebral; entretanto, ainda não foram comprovados os benefícios dessas medidas na prevenção de recorrência de crises de déficits neurológicos transitórios. Para a clínica de hipertensão intracraniana, alguns autores defendem a ideia do uso de glicocorticoides na abordagem do evento, supondo-se a natureza inflamatória do processo, porém, sem eficácia comprovada. Outras estratégias de redução da pressão intracraniana podem ser usadas, como uso de acetazolamida, ${ }^{(43)}$ que atua por meio de mecanismo não dependente de inflamação, diminuindo fundamentalmente a produção de LCR.

Não se deve esquecer que a síndrome está intrinsecamente ligada a uma condição álgica, sendo a abordagem da cefaleia, que na maioria das vezes é de moderada a forte intensidade, de fundamental importância na terapêutica, lançando mão de medicações abortivas e, caso necessário, adoção de medidas profiláticas.

\section{Prognóstico}

Na grande maioria dos casos, o prognóstico da síndrome é excelente, apresentando resolução completa das alterações clínicas e do LCR em cerca de três meses. Podem ocorrer recorrências dos episódios de défices neurológicos neste período, contudo, a persistência prolongada de sequelas neurológicas deve levar a outras hipóteses diagnósticas, já que esta condição, por definição, possui caráter autolimitado.

\section{CONCLUSÃO}

O diagnóstico diferencial entre HaNDL e migrânea hemiplégica familiar pode ser difícil, já que ambas pactuam em algumas características. O histórico de múltiplas crises em curto intervalo de tempo, o sexo masculino e a idade compatível são fatores que corroboraram a hipótese de HaNDL. É importante destacar a importância do estudo do LCR para se realizar a diferenciação entre estas duas entidades, iá que a linfocitose é característica da HaNDL. O conhecimento sobre esta doença é importante para que se aumente o grau de suspeição em pacientes que apresentam quadros clínicos compatíveis e já foram intensamente investigados e não apresentaram resultados conclusivos, reduzindo assim o tempo de internamento e a realização de procedimentos invasivos desnecessários. $\bigcirc$ esclarecimento sobre o caráter muitas vezes benigno e autolimitado da doença pode contribuir para diminuir o grau de ansiedade nos pacientes e em seus familiares.

\section{REFERÊNCIAS}

1. Hutton J, Wellington D, Miller S. HaNDL syndrome: transient headache and neurological deficits with cerebrospinal fluid lymphocytosis. N Z Med J 2017;130(1449):67-9.

2. Valenca MM, de Oliveira DA, Martins HA. Alice in Wonderland Syndrome, Burning Mouth Syndrome, Cold Stimulus Headache, and HaNDL: Narrative Review. Headache 2015; 55(9): 1233-48.

3. Nakashima K. Syndrome of transient headache and neurological deficits with cerebrospinal fluid lymphocytosis: HaNDL. Intern Med 2005;44(7):690-1.

4. Gomez-Aranda F, Canadillas F, Marti-Masso JF, Diez-Tejedor E, Serrano PJ, Leira $R$, et al. Pseudomigraine with temporary neurological symptoms and lymphocytic pleocytosis. A report of 50 cases. Brain. 1997; 120 ( Pt 7): $1105-13$.

5. Bartleson JD, Swanson JW, Whisnant JP. A migrainous syndrome with cerebrospinal fluid pleocytosis. Neurology. 1981;31(10): 1257-62.

6. Filina T, Feja KN, Tolan RW, Jr. An adolescent with pseudomigraine, transient headache, neurological deficits, and lymphocytic pleocytosis (HaNDL Syndrome): case report and review of the literature. Clin Pediatr (Phila). 2013;52(6):496-502.

7. Verentzioti A, Tavernarakis A, Mamali M, Siatouni A, Gatzonis $S$. Pseudomigraine with transient neurological deficits and cerebrospinal fluid lymphocytosis or HaNDL syndrome: A case report with confusion and positive IgM antibodies to CMV in serum. Cephalalgia. 2017;37(1):99-100.

8. Fumal A, Vandenheede M, Coppola G, Di Clemente L, Jacquart J, Gerard P, et al. The syndrome of transient headache with neurological deficits and CSF lymphocytosis (HaNDL): electrophysiological findings suggesting a migrainous pathophysiology. Cephalalgia. 2005;25(9):754-8.

9. The International Classification of Headache Disorders, 3rd edition (beta version). Cephalalgia. 2013;33(9):629-808.

10. Burke MJ, Lamb MJ, Hohol M, Lay C. Unique CT Perfusion Imaging in a Case of HaNDL: New Insight into HaNDL Pathophysiology and Vasomotor Principles of Cortical Spreading Depression. Headache. 2017;57(1):129-34.

11. Raets I. Diffusion restriction in the splenium of the corpus callosum in a patient with the syndrome of transient headache with neurological deficits and CSF lymphocytosis (HaNDL): a challenge to the diagnostic criteria? Acta Neurol Belg. 2012; $112(1): 67-9$.

12. Segura T, Hernandez-Fernandez F, Sanchez-Ayaso P, Lozano E, Abad L. Usefulness of multimodal MR imaging in the differential diagnosis of HaNDL and acute ischemic stroke. BMC Neurol. 2010;10:120.

13. Vallet AE, Desestret $V$, Tahon F, Cho TH, Nighoghossian N. Acute perfusion MR imaging in a HaNDL-like syndrome. Cerebrovasc Dis. 2010;29(1):98-100.

14. Yilmaz A, Kaleagasi H, Dogu O, Kara E, Ozge A. Abnormal $M R I$ in a patient with 'headache with neurological deficits and CSF lymphocytosis (HaNDL)'. Cephalalgia. 2010;30(5): 615-9.

15. Bicakci S, Kurtaran B, Over MF, Bicakci YK. Are the Comments on HaNDL Syndrome in the ICHD-II Sufficient? Noro Psikiyatr Ars. $2014 ; 51(2): 178-80$. 
16. Hidalgo de la Cruz M, Dominguez Rubio R, Luque Buzo E, Diaz Otero F, Vazquez Alen P, Orcajo Rincon J, et al. Syndrome of transient headache and neurological deficits with cerebrospinal fluid lymphocytosis (HaNDL) in a patient with confusional symptoms, diffuse EEG abnormalities, and bilateral vasospasm in transcranial Doppler ultrasound: A case report and literature review. Neurologia. 2017 Apr 17. pii: S0213-4853(17) 301469. [Article in English, Spanish].

17. Quintas S, Lopez Ruiz R, Trillo S, Gago-Veiga AB, ZapataWainberg G, Dotor Garcia-Soto J, et al. Clinical, imaging and electroencephalographic characterization of three cases of HaNDL syndrome. Cephalalgia. 2018;38(7):1402-06.

18. Kappler J, Mohr S, Steinmetz H. Cerebral vasomotor changes in the transient syndrome of headache with neurologic deficits and CSF lymphocytosis (HaNDL). Headache. 1997;37(8):516-8.

19. Baron J, Mulero P, Pedraza MI, Gamazo C, de la Cruz C, Ruiz $M$, et al. HaNDL syndrome: Correlation between focal deficits topography and EEG or SPECT abnormalities in a series of 5 new cases. Neurologia. 2016;31 (5):305-10.

20. Stelten BM, Venhovens J, van der Velden LB, Meulstee J, Verhagen WI. Syndrome of transient headache and neurological deficits with cerebrospinal fluid lymphocytosis (HaNDL): A case report with serial electroencephalography (EEG) recordings. Is there an association with human herpes virus type 7 (HHV-7) infection? Cephalalgia. 2016;36(13):1296-301.

21. Tsang BK, Kwong JC, Dewey HM. Case of syndrome of headache with neurological deficits and cerebrospinal fluid lymphocytosis (HaNDL) with focal slowing on electroencephalogram. Intern Med J. 2012;42(8):944-7.

22. Anagnostou E, Spengos K, Naoumis D, Paraskevas GP, Vassilopoulou S, Zis V, et al. Lack of visual evoked potential habituation in the syndrome of HaNDL. J Neurol. 2009;256 (8):1374-6.

23. Berthold $\bigcirc$, Theophil M, von Moers A. HaNDL Syndrome with Fever in a 12-Year-Old Boy - A Case Report. Headache. 2018; 58(4):597-98.

24. Emond H, Schnorf H, Poloni C, Vulliemoz S, Lalive PH. Syndrome of transient headache and neurological deficits with CSF lymphocytosis (HaNDL) associated with recent human herpesvirus-6 infection. Cephalalgia. 2009;29(4):487-91.

25. Kurtuncu M, Kaya D, Zuliani L, Erdag E, Icoz S, Ugurel E, et al. CACNA1H antibodies associated with headache with neurological deficits and cerebrospinal fluid lymphocytosis (HaNDL). Cephalalgia. 2013;33(2): 123-9.

26. Erdag E, Celebisoy N, Yuceyar AN, Kurtuncu M, Vural B, Tuzun E. Antibodies to DNA repair proteins in headache with neurological deficits and cerebrospinal fluid lymphocytosis (HaNDL) patients. Acta Neurol Belg. 2015; 1 15(2):137-40.

27. Lo Re M, di Sapio A, Malentacchi M, Granieri L, Bertolotto A. Acute confusional state in $\mathrm{HaNDL}$ syndrome (transient headache and neurologic deficits with cerebrospinal fluid lymphocytosis). Neurol Sci. 2015;36(3):477-8.

28. Martinez-Velasco E, Mulero P, Baron J, Amer M, Guerrero AL. Confusional state in $\mathrm{HaNDL}$ syndrome: an uncommon clinical manifestation. Neurol Sci. 2016;37(3):483-5.

29. Nelson S. Confusional State in HaNDL Syndrome: Case Report and Literature Review. Case Rep Neurol Med 2013;2013: 317685
30. Parissis D, loannidis P, Balamoutsos G, Karacostas D. Confusional state in the syndrome of HaNDL. Headache. $2011 ; 51$ (8): $1285-8$

31. Samanta D, Willis E. Rapid improvement of the confusional state and electroencephalography after spinal tap in a patient with headache and neurologic deficits with cerebrospinal fluid lymphocytosis (HaNDL) syndrome. Neurol India. 2015;63(6): 978-9.

32. Chan JW, Cheng C. Complete external ophthalmoplegia in headache, neurologic deficits, and cerebrospinal fluid lymphocytosis (HaNDL) syndrome. Eye (Lond). 2010;24(1): 198-9.

33. Gungor I, Cakar A, Kocasoy Orhan E, Baykan B. [A HaNDL case with papilledema mimicking transient ischemic attack]. Agri $2016 ; 28(4): 199-202$.

34. Cifelli A, Vaithianathar L. Syndrome of transient Headache and Neurological Deficits with cerebrospinal fluid Lymphocytosis (HaNDL). BMJ Case Rep. $2011 ; 2011$.

35. Armiento R, Kornberg AJ. Altered conscious state as a presentation of the syndrome of transient headache and neurological deficits with cerebrospinal fluid lymphocytosis ( $\mathrm{HaNDL}$ syndrome) in a paediatric patient. J Paediatr Child Health. 2016;52(7):774-6.

36. Goncalves D, Meireles J, Rocha R, Sampaio M, Leao M. Syndrome of transient headache and neurologic deficits with cerebrospinal fluid lymphocytosis (HaNDL): a pediatric case report. J Child Neurol. 2013;28(12):1661-3.

37. Rivero-Sanz E, Pias-Peleteiro L, Gonzalez-Alvarez V. HaNDL syndrome in a 14-year-old girl. BMJ Case Rep 2016;2016.

38. Frediani F, Bussone G. Confusional state as first symptom of HaNDL syndrome. Neurol Sci. 2015;36 Suppl 1:71-4.

39. Finke C, Mengel A, Pruss H, Stocker W, Meisel A, Ruprecht K. Anti-NMDAR encephalitis mimicking HaNDL syndrome. Cephalalgia. 2014;34(12):1012-4.

40. Fernandez N, Wijeyekoon R, Richardson A, Jones M. HaNDL with care. Acute Med. 2015;14(3):119-21.

41. Gomez-Alonso J, Munoz-Garcia D, Rodriguez-Rodriguez M. [HaNDL syndrome and Hashimoto's encephalopathy]. Rev Neurol. 2008;46(4):255-6.

42. Krause T, Nolte $\mathrm{CH}$. The syndrome of transient headache and neurological deficits with cerebrospinal fluid lymphocytosis ( $\mathrm{HaNDL}$ ) as an acute ischemic stroke mimic leading to systemic thrombolysis: a case report. Clin Neurol Neurosurg. 2012;114 (6):689-90.

43. Mulroy E, Yap J, Danesh-Meyer H, Anderson N. Symptomatic intracranial hypertension during recovery from the syndrome of headache with neurologic deficits and cerebrospinal fluid lymphocytosis (HANDL). Pract Neurol. 2017;17(2): 145-48.

\section{Correspondência}

Laryssa Crystinne Azevedo Almeida, E-mail: laryssazevedo@hotmail.com

Recebido: 2 de maio de 2018

Aceito: 15 de maio de 2018 